\title{
ON EMBEDDABLE FINITE AMALGAMS OF GROUPS
}

\author{
by ABDUL MAJEED
}

(Received 12 October, 1970)

1. In this paper, a problem of B. H. Neumann and Hanna Neumann [7] about the finite embeddability of an embeddable finite amalgam is discussed. After proving a "reduction theorem" for a finite amalgam to have a finite embedding, we examine some known embeddable amalgams (cf. [3]) as regards their embeddability in a finite group. Since the existence of the generalised free product and the embeddability of an amalgam are synonymous terms, Theorem 3.1 generalises a result in [4]. A sufficient condition for an amalgam of type $S$ to have a finite embedding is also given.

In $\S 4$, an example is given to answer various questions regarding the range of finite embeddings of a finite amalgam of three groups.

The definition and properties of amalgams and their generalised free products may be found in [7], [8], [4] and [6].

2. Let $\mathrm{A}$ be an amalgam of groups $G_{1}, G_{2}, \ldots, G_{n}$ with amalgamated subgroups $H_{i j}$ $(i, j=1,2, \ldots, n ; i \neq j)$ and let $\mathrm{A}^{\prime}$ be their reduced amalgam. This is the amalgam formed by the groups $H_{i}$ generated by all the $H_{i j}$ (i fixed, $j \neq i$ ). It is known that the generalised free product of $A$ exists if and only if that of $A^{\prime}$ exists (cf. [4], [6]). We prove here a similar reduction theorem.

THEOREM 2.1. A finite amalgam A of the groups $G_{1}, G_{2}, \ldots, G_{n}$ amalgamating $H_{i j}$ is embeddable in a finite group if and only if their reduced amalgam $\mathrm{A}^{\prime}$ is so embeddable.

Proof. The necessity of the condition is immediate. To prove that this condition is also sufficient, let $H$ be a finite embedding of the reduced amalgam $\mathrm{A}^{\prime}$. Then, for any integer $i(i=1,2, \ldots, n)$, the groups $G_{i}$ and $H$ both contain a subgroup $H_{i}$ generated by all the $H_{i j}$ ( $i$ fixed, $j \neq i$ ), and their amalgam is embeddable in a finite group $K_{i}$, say, (cf. [9], [8]). Each such $K_{i}$ contains the finite group $H$ as a subgroup and their amalgam has a finite embedding, namely their permutational product [9].

In view of the above theorem, we need examine the finite embeddability of only the reduced amalgam. Such an embedding of the reduced amalgam will ensure that of the whole amalgam as well.

We now make a few observations about an amalgam of three finite groups. To my knowledge little is known as regards the finite embeddings of such amalgams. Because of the difficulties involved in knowing whether such an amalgam is even embeddable or not, the problem of finding a finite embedding becomes even more complicated. Some results concerning the existence of generalised free product of certain special types of amalgams of three groups were given in [3]. It was observed that in these cases the embedding groups, namely the generalised free products, were finite. It can also be seen [8, example II.1] that an amalgam of three groups $A, B, C$ generated by the subgroups $K, L ; K, M$ and $L, M$ with 
$B$ and $C$ as split extensions of $K$ by $M$ and of $L$ by $M$, respectively, need not be embeddable. However, if such an amalgam is embeddable, then it has a finite embedding. The proof of the above remarks is left to the reader.

3. Let A be an " amalgam of type $S$ ". This is an amalgam of $n$ groups $G_{1}, G_{2}, \ldots, G_{n}$ arranged in such a manner that

$$
G_{i} \cap G_{i+1}=H_{i}, \quad G_{i} \cap G_{J}=H \quad(j \neq i \pm 1), \quad G_{n+1}=G_{n}, \quad H_{n+1}=H_{1} .
$$

In the reduced amalgam, each $G_{i}$ can be taken as a group generated by $H_{i-1}$ and $H_{i}$. The existence of the generalised free product of such an amalgam was established in [3]. It is not known whether such an amalgam has a finite embedding also. For $n=3$, some sufficient conditions for the finite embeddability of this type of amalgams were given in [6]. The following theorem mentions one such sufficient condition for the embeddability of amalgam of type $S$ in a finite group.

THEOREM 3.1. Let $\mathrm{A}$ be a finite amalgam of $n$ groups of type $S, n>3$ and put $m=\left[\frac{1}{2} n\right]$. If $G_{m+1}, G_{m+2}, \ldots, G_{n}$ have the property that, in each $G_{i}, H_{i-1}$ and $H_{i}$ permute elementwise and further that $H_{i} \cap H_{j}=H$ is central in $G_{i}, G_{j}$, for all $i, j=1,2, \ldots, n$, then $\mathrm{A}$ is embeddable in a finite group.

The proof of this follows in a fashion similar to the one given for the existence of the generalised free product of an amalgam of type $S$ in [3], using the direct product with a single amalgamation in place of the free product.

Hanna Neumann has shown (cf. [4, p. 623]), that, in the case of the most general type of an amalgam of $n$ groups, with $n>3$, if $n-1$ of the groups have the property that their subgroups $H_{\text {in }}$ ( $i$ fixed) commute elementwise, the generalised free product of this amalgam may not exist. However, for the particular amalgam of type $S$ mentioned above, which reduces to that in Theorem 9.1 of [4], for $n=3$, such a result and even a slightly more general version of it is seen to be true.

Another sufficient condition for the finite embeddability of an amalgam of type $S$ is given in the following theorem.

THEOREM 3.2. Let the finite groups $G_{1}, G_{2}, \ldots, G_{n}$ form a reduced amalgam A of type $S$. Let further $H_{1}, H_{n-1}$ be normal subgroups of $G_{1}$ and $G_{n}$ respectively, and $H=G_{i} \cap G_{j}(j \neq i \pm 1)$ be central in $G_{i}(i=1,2, \ldots, n)$. Then $\mathrm{A}$ is embeddable in a finite group if, when $n=2 m$, $H_{m-1}$ and $H_{m+1}$ are normal in $G_{m}$ and $G_{m+1}$ respectively, and when $n=2 m+1, G_{m+1}$ is such that its subgroups $H_{m}$ and $H_{m+1}$ are permutable elementwise.

Proof. To begin with we note that, for $n=3$, the generalised free product of the amalgam exists and is finite by Theorem 3.1 of [3]. For $n>3$, we write, as in Theorem 5.1 of [3], the groups $G_{1}, G_{2}, \ldots, G_{n}$ in pairs $\left(G_{i}, G_{2 m-i+1}\right)$ or $\left(G_{i}, G_{2 m-i+2}\right)$, respectively, according as $n=2 m$ or $n=2 m+1$, leaving $G_{m+1}$ alone in the second case.

In the first event, $H_{1}$ is normal in $G_{1}$ and $H_{n-1}$ in $G_{n}$, and $G_{1}, G_{n}$ are generated by $H_{n}, H_{1}$ and $H_{n-1}, H_{n}$ respectively. By Lemma $P$ of [6], the permutational product $P_{1}$ of $G_{1}, G_{n}$ amalgamating $H_{n}$ with transversals $H_{1}^{\prime}, H_{n-1}^{\prime}$, where $H_{1}^{\prime} \cdot H=H_{1}, H_{n-1}^{\prime} \cdot H=H_{n-1}$, is such that the groups $H_{1}=\rho\left(H_{1}\right), H_{n-1}=\rho\left(H_{n-1}\right)$ in $P_{1}$ generate their generalised direct product 
$\left(H_{1} \times H_{n-1} ; H\right)$ amalgamating a central subgroup $H$. Similarly for the amalgam of $G_{m}, G_{m+1}$. Since $H$ is central in every $G_{i}$ and $G_{i} \cap G_{2 m-i+1}=H(i=2,3, \ldots, m-1)$, the generalised direct product $P_{i}$ of $G_{i}, G_{2 m-i+1}$ amalgamating $H\left(P_{i}\right.$ is also their permutational product [9]) exists for every $i$. Each such $P_{i}$ is finite and has the following property.

Whenever $H_{i}$ and $H_{i+1}$ belong to such a permutational product, they generate in it the group $G_{i+1}$, and whenever $H_{i}, H_{j}, j \neq i \pm 1$, belong to it, they generate in it their generalised direct product amalgamating $H$.

Now $P_{1}=\mathrm{gp}\left(H_{1}, H_{2 m-1}, H_{2 m}\right)$ and $P_{2}=\mathrm{gp}\left(H_{1}, H_{2 m-2}, H_{2 m-1}\right)$ have a common subgroup $\left(H_{1} \times H_{2 m-1} ; H\right)$. There exists a finite embedding $P^{(1)}$ of $P_{1}$ and $P_{2}$ amalgamating this subgroup. $P^{(1)}$ then contains the groups $G_{1}, G_{2}, G_{2 m-1}$ and $G_{2 m}$. Since $P_{3}=\operatorname{gp}\left(H_{2}, H_{3}\right.$, $\left.H_{2 m-3}, H_{2 m-2}\right)$ contains the group $\left(H_{2} \times H_{2 m-2} ; H\right)$ which is also a subgroup of $P_{2}$ and hence of $P^{(1)}$, a finite embedding $P^{(2)}$ of $P^{(1)}$ and $P_{3}$ amalgamating $\left(H_{2} \times H_{2 m-2} ; H\right)$ can be found. Continuing in this way, we see that there is a finite embedding $P^{(m-2)}$ of $P^{(m-3)}$ and $P_{m-1}$ amalgamating $\left(H_{m-2} \times H_{m+2} ; H\right)$. In $P^{(m-2)}$, the groups $H_{m-1}$ and $H_{m+1}$ commute elementwise as shown above. Therefore a permutational product $P^{(m-1)}$ of $P^{(m-2)}$ and $P_{m}$ amalgamating $\left(H_{m-1} \times H_{m+1} ; H\right)$ embeds the amalgam of $P^{(m-2)}$ and $P_{m} . \quad P^{(m-1)}$ obviously contains the groups $G_{1}, G_{2}, \ldots, G_{n}$.

When $n=2 m+1$, we form the generalised direct products $P_{i}$ of $G_{i}$ and $G_{2 m-i+2}$ amalgamating a central subgroup $H(i=2,3, \ldots, m)$ and also the permutational product $P_{1}$ of $G_{1}, G_{n}$ amalgamating $H_{n}$ using transversals $H_{1}^{\prime}, H_{n-1}^{\prime}, H_{1}^{\prime} H=H_{1}^{\prime}, H_{n-1}^{\prime} H=H_{n-1}$. In $P_{1}$, the groups $H_{1}, H_{n-1}$ generate their generalised direct product amalgamating $H$. We now construct, as above, a finite group $P^{(m-1)}$ which is an embedding of the finite amalgam of $P^{(m-2)}$ and $P_{m}$ amalgamating $\left(H_{m-2} \times H_{m+2} ; H\right)$. However, as $m>1, G_{m}, G_{m+2}$ have only $H$ in common and $P_{m}$ is the generalised direct product of these groups. Hence $G_{m+1}=$ $\left(H_{m} \times H_{m+1} ; H\right)$ is a subgroup of $P_{m}$ and so also of $P^{(m-1)}$. Thus in this case again $P^{(m-1)}$ contains all the $G_{i}$ 's $(i=1,2, \ldots, n)$. That they have their right intersection in $P^{(m-1)}$ follows by an argument similar to the one in Theorem 5.1 of [3]. This completes the proof of the theorem.

This is as far as one could hope to go in the general case. Things look more obscure when we do not have any restriction on the constituents. The main difficulty lies with the amalgam of three finite groups. If such an amalgam is embeddable in a finite group, it is easy to prove that a finite amalgam of type $S$ has a finite embedding.

4. In this section we construct an example to answer the following questions regarding the range of finite embeddings of a finite amalgam of three groups.

(i) Is there an infinite embedding of three finite groups $A, B, C$ other than the free one? If so, is there also a finite embedding of $A, B, C$ ?

(ii) Let $P$ and $P^{\prime}$ be two finite embeddings of the groups $A, B$ and $C$ such that the order of $P$ is larger than that of $P^{\prime}$. Does there always exist a homomorphism of $P$ onto $P^{\prime}$ ?

(iii) Is there always a unique minimal embedding of an amalgam of three finite groups?

These questions may be answered by interpreting groups described by Coxeter and Moser as generalised free products. 
Example. Let $(l, m, n ; p)$ denote the group generated by $S$ and $T$ having the defining relations

$$
S^{l}=T^{m}=(S T)^{n}=\left(S T^{-1} S^{-1} T\right)^{p}=1 .
$$

When $n=2$, so that

$$
S T=(S T)^{-1}=T^{-1} S^{-1},
$$

we have

$$
S T^{-1} S^{-1} T=S S T T=S^{2} T^{2}
$$

Hence $(l, m, 2 ; p)$ is also defined by

$$
S^{l}=T^{m}=(S T)^{2}=\left(S^{2} T^{2}\right)^{p}=1 .
$$

If $l=5, m=5, p=3$, then the group $(5,5,2 ; 3)$ has a representation as

$$
S^{5}=T^{5}=(S T)^{2}=\left(S^{2} T^{2}\right)^{3}=1 .
$$

The infiniteness of $(5,5,2 ; 3)$ follows by Coxeter $[1$, p. 93].

The amalgam we construct is such that its generalised free product shall have as a proper homomorphic image a group containing a subgroup isomorphic to $(5,5,2 ; 3)$. The three groups we take are the dihedral groups of orders 4,10 and 10 such that the amalgamated subgroup of each pair is a cyclic group of order 2 . Thus

$$
\begin{aligned}
& A=\operatorname{gp}\left(a, b ; a^{2}=b^{2}=(a b)^{2}=1\right), \\
& B=\operatorname{gp}\left(b, c ; b^{2}=c^{2}=(b c)^{5}=1\right), \\
& C=\operatorname{gp}\left(c, a ; c^{2}=a^{2}=(c a)^{5}=1\right) .
\end{aligned}
$$

The free product of $A, B, C$ is

$$
F=\operatorname{gp}\left(a, b, c ; a^{2}=b^{2}=c^{2}=(b c)^{5}=(c a)^{5}=(a b)^{2}=1\right),
$$

which can, following Coxeter and Moser [2], also be considered as a group generated by reflections " $a$ ", " $b$ ", " $c$ " in the sides of a spherical triangle with angles $\pi / 5, \pi / 5$ and $\pi / 2$ respectively. $F$ can also be represented as

$$
F=\operatorname{gp}\left(g, h, c ; g^{5}=h^{5}=(g h)^{2}=(g c)^{2}=(c h)^{2}=c^{2}=1\right),
$$

where $b c=g, c a=h$, so that $b a=g h$. Since

$$
g^{c}=c(b c) c=c b=(b c)^{-1}=g^{-1}
$$

and

$$
h^{c}=c(c a) c=a c=(c a)^{-1}=h^{-1}
$$

the subgroup

$$
G_{1}=\mathrm{gp}\left(g, h ; g^{5}=h^{5}=(g h)^{2}=1\right)
$$

of $F$ is normal in $F$. Moreover, since $G_{1} \cap \mathrm{gp}(c)=1, F$ is a split extension of $G_{1}$ by a cyclic group of order 2. Further, $G_{1}$ is an infinite group by Coxeter [2, p. 54]. 
Let $N$ be the normal closure of $\left(g^{2} h^{2}\right)^{3}$ in $G_{1}$. We show that the normal closure $N^{*}$ of $N$ in $F$ coincides with $N$. For this we have only to prove that

$$
c^{-1}\left(g^{2} h^{2}\right)^{3} c
$$

is an element of $N$. Now

$$
c^{-1}\left(g^{2} h^{2}\right)^{3} c=\left(g^{-2} h^{-2}\right)^{3}=\left(h^{2} g^{2}\right)^{-3}
$$

and, since

$$
g^{-2}\left(g^{2} h^{2}\right)^{3} g^{2}=g^{-2} g^{2} h^{2} g^{2} h^{2} g^{2} h^{2} g^{2}=\left(h^{2} g^{2}\right)^{3}
$$

belongs to $N$, therefore $c^{-1}\left(g^{2} h^{2}\right)^{3} c$, being the inverse of $\left(h^{2} g^{2}\right)^{3}$, is also in $N$; this shows that $N$ is also normal in $F$. If we denote the factor group $F / N$ by $F_{1}$, then

$$
F_{1}=\operatorname{gp}\left(g^{\prime}, h^{\prime}, c^{\prime} ; g^{\prime 5}=h^{\prime 5}=\left(g^{\prime} h^{\prime}\right)^{2}=\left(g^{\prime 2} h^{\prime 2}\right)^{3}=\left(g^{\prime} c^{\prime}\right)^{2}=\left(c^{\prime} h^{\prime}\right)^{2}=c^{\prime 2}=1\right),
$$

or, on identification of $g^{\prime}, h^{\prime}, c^{\prime}$ with $g, h, c$ respectively,

$$
F_{1}=\operatorname{gp}\left(g, h, c ; g^{5}=h^{5}=(g h)^{2}=\left(g^{2} h^{2}\right)^{3}=(g c)^{2}=(c h)^{2}=c^{2}=1\right) .
$$

However the group

$$
G_{2}=\operatorname{gp}\left(g, h ; g^{5}=h^{5}=(g h)^{2}=\left(g^{2} h^{2}\right)^{3}=1\right)
$$

is a normal subgroup of $F_{1}$ and, since $G_{2} \cap \operatorname{gp}(c)=1, F_{1}$ is a splitting extension of $G_{2}$ by a two-cycle. $G_{2}$, being the same as the group $(5,5,2 ; 3)$, is infinite and so also is, therefore, $F_{1}$. In terms of $a, b, c, F_{1}$ has the presentation

$$
F_{1}=\operatorname{gp}\left(a, b, c ; a^{2}=b^{2}=c^{2}=(b c)^{5}=(c a)^{5}=(a b)^{2}=(b c a)^{6}=1\right) .
$$

Since every element of $B$ and $C$ is of the form

$$
(b c)^{k} b^{r} \text { and }(c a)^{k^{\prime}} c^{r^{\prime}}
$$

respectively, $\left(k, k^{\prime}=0,1,2, \ldots, 4 ; r, r^{\prime}=0,1\right)$, and since for no nonzero values of $k$ and $k^{\prime}$ is the relation $(b c)^{k} b^{r}=(c a)^{k^{\prime}} c^{r^{\prime}}$ implied by the additional relation $(b c a)^{6}=1$, therefore there are no additional amalgamations of $B$ and $C$ and similarly none of $C$ and $A$ or of $A$ and $B$. The groups $A, B, C$ have, therefore, their precise intersections in $F_{1}$ and $F_{1}$ consequently embeds their amalgam.

However, $F_{1}$ is different from the free embedding $F$ of the amalgam of $A, B, C$ and moreover, is infinite. This answers the first part of question (i).

We now show that there is also a finite embedding of the amalgam of $A, B, C$. Consider the group

$$
G_{3}=\mathrm{gp}\left(g^{\prime}, h^{\prime} ; g^{\prime 5}=h^{\prime 5}\left(g^{\prime} h^{\prime}\right)^{2}=\left(g^{\prime-1} h^{\prime}\right)^{3}=1\right) .
$$

$G_{3}$ is isomorphic to the smallest nonabelian simple group $A_{5}$ of even permutations on five letters (cf. Coxeter [2]). However, a factor group of $G_{1}$ determined by the normal closure $N^{\prime}$ of $\left(g^{-1} h\right)^{3}$ in $G_{1}$ is also isomorphic to $G_{3}$. We prove that $N^{\prime}$ is a normal subgroup of $F$.

Since $g=b c, h=c a$, and

$$
a^{-1}\left(g^{-1} h\right) a=a c b c a a=a c b c=h^{-1} g=\left(g^{-1} h\right)^{-1},
$$

we have $a^{-1}\left(g^{-1} h\right)^{3} a=\left(g^{-1} h\right)^{-3} \in N^{\prime}$. 
Further, since

$$
b^{-1} g^{-1} h b=b c b c a b=g^{2} \cdot(g h)^{-1}=g^{2} h^{-1} g^{-1},
$$

we have

$$
\begin{aligned}
b^{-1}\left(g^{-1} h\right)^{3} b & =g^{2} h^{-1} g^{-1} g^{2} h^{-1} g^{-1} g^{2} h^{-1} g^{-1} \\
& =g^{2} h^{-1} g \cdot h^{-1} g \cdot h^{-1} g \cdot g^{-2} \\
& =g^{2}\left(h^{-1} g\right)^{3} g^{-2} \\
& =g^{2}\left(g^{-1} h\right)^{-3} g^{-2} \in N^{\prime} .
\end{aligned}
$$

Also

$$
c^{-1} g^{-1} h c=c c b c a c=b c \cdot a c=g h^{-1} .
$$

Thus

$$
\begin{aligned}
c^{-1}\left(g^{-1} h\right)^{3} c & =g h^{-1} g h^{-1} g h^{-1}=g \cdot h^{-1} g h^{-1} g h^{-1} g \cdot g^{-1} \\
& =g\left(h^{-1} g\right)^{3} g^{-1}=g\left(g^{-1} h\right)^{-3} g^{-1} \in N^{\prime},
\end{aligned}
$$

showing that $N^{\prime}$ is a normal subgroup of $F$. Therefore

$$
F / N^{\prime}=\operatorname{gp}\left(G_{1} / N^{\prime}, c^{\prime}\right)=\operatorname{gp}\left(G_{3}, c^{\prime}\right)=F_{2}, \text { say. }
$$

Again identifying $g^{\prime}, h^{\prime}, c^{\prime}$ with $g, h, c$, respectively, we have

$$
F_{2}=\operatorname{gp}\left(g, h, c ; g^{5}=h^{5}=(g h)^{2}=\left(g^{-1} h\right)^{3}=(g c)^{2}=(c h)^{2}=c^{2}=1\right)
$$

and in terms of $a, b, c$,

$$
F_{2}=\mathrm{gp}\left(a, b, c ; a^{2}=b^{2}=c^{2}=(b c)^{5}=(c a)^{5}=(a b)^{2}=(c b c a)^{3}=1\right) .
$$

By a reasoning similar to that above, we see that also in $F_{2}, A, B, C$ have their precise intersections. $F_{2}$, therefore, embeds the amalgam of $A, B, C$. The finiteness of $F_{2}$ follows from the fact that it is an extension of a finite group $G_{3}$ by a cyclic group of order 2 . This completes the answer to the first question.

We now show that there exist two finite embeddings with the property that the smaller embedding is not a homomorphic image of the larger one. We consider the group

$$
G_{4}=g p\left(g^{\prime \prime}, h^{\prime \prime} ; g^{\prime \prime 5}=h^{\prime \prime 5}=\left(g^{\prime \prime} h^{\prime \prime}\right)^{2}=\left(g^{\prime \prime-1} h^{\prime \prime}\right)^{4}=1\right),
$$

which is isomorphic to the simple group $A_{6}$ of even permutations on six letters (Coxeter [8], p. 79), and is a homomorphic image of $G_{1}$, where $G_{1}$ is the same as in (1). The kernel $N^{\prime \prime}$ of this homomorphism is the normal closure of $\left(g^{-1} h\right)^{4}$ in $G_{1}$. We show again that $N^{\prime \prime}$ is a normal subgroup of $F$. It is enough to show that the transforms of $\left(g^{-1} h\right)^{4}$ by $a, b, c$ are in $N^{\prime \prime}$. Now

$$
\begin{aligned}
a^{-1} g^{-1} h a & =\left(g^{-1} h\right)^{-1}, \\
b^{-1}\left(g^{-1} h\right) b & =g^{2} h^{-1} g, \\
c\left(g^{-1} h\right) c & =g h^{-1}
\end{aligned}
$$


and therefore, since, as before

$$
a\left(g^{-1} h\right)^{4} a=\left(g^{-1} h\right)^{-4}, \quad b\left(g^{-1} h\right)^{4} b=g^{2}\left(g^{-1} h\right)^{-4} g^{-2} \quad \text { and } \quad c\left(g^{-1} h\right)^{4} c=g\left(g^{-1} h\right)^{-4} g^{-1}
$$

are elements of $N^{\prime \prime}, N^{\prime \prime}$ is a normal subgroup of $F$ and the factor group

$$
F_{3}=F / N^{\prime \prime}=\mathrm{gp}\left(G_{4}, c^{\prime}\right),
$$

being a split extension of $G_{4}$ by $\mathrm{gp}(c)$, is finite.

In terms of $a, b, c, F_{3}$ is given by

$$
F_{3}=\operatorname{gp}\left(a, b, c ; a^{2}=b^{2}=c^{2}=(b c)^{5}=(c a)^{5}=(a b)^{2}=(c b c a)^{4}=1\right) .
$$

That $F_{3}$ embeds the amalgam of $A, B$ and $C$ follows as before. However, considering

$$
F_{3}=\operatorname{gp}\left(G_{4}, c^{\prime \prime}\right) \text { and } F_{2}=\operatorname{gp}\left(G_{3}, c^{\prime}\right),
$$

one sees that $F_{2}$ cannot be homomorphic image of $F_{3}$, because of the simplicity of $G_{3}$ and $G_{4}$. This answers question (ii).

Moreover $F_{2}$ and $F_{3}$ are minimal finite embeddings of $A, B, C$ in the sense that there are no proper factor groups of $F_{2}$ and $F_{3}$ which embed the amalgam of $A, B, C$. This shows that the answer to question (iii) is in the negative.

The following problem remains unsolved: If the generalised free product $F$ of a finite amalgam is finite, can there exist another finite embedding of the amalgam different from $F$ ?

Finally, I should like to thank Professor Hanna Neumann for her valuable suggestions and constructive criticism in the preparation of this paper.

\section{REFERENCES}

1. H. S. M. Coxeter, The abstract groups $G^{m, n, p}$, Trans. Amer. Math. Soc. 45 (1939), 73-150. 1957).

2. H. S. M. Coxeter and J. Moser, Generators and relations for discrete groups (Springer-Verlag,

3. A. Majeed, Permutational products of groups and embedding theory of group amalgams, M.A. Thesis presented to the Australian National University (1966).

4. H. Neumann, Generalised free products of groups with amalgamated subgroups I, Amer. J. Math. 70 (1948), 590-625.

5. H. Neumann, Generalised free products of groups with amalgamated subgroups II, Amer. J. Math. 71 (1949), 491-540.

6. B. H. Neumann and Hanna Neumann, A remark on generalised free products, $J$. London Math. Soc. 25 (1950), 202-204.

7. B. H. Neumann and Hanna Neumann, A contribution to the embedding theory of group amalgams, Proc. London Math. Soc. (3) 3 (1953), 245-256.

8. B. H. Neumann, An essay on free products of groups with amalgamations, Philos. Trans. Roy. Soc. London, Ser. A 246 (1954), 503-554.

9. B. H. Neumann, Permutational products of groups, J. Australian Math. Soc. 1 (1960), 299-310.

University of The PunJab

LAHORE, PAKISTAN 